\title{
AVALIAÇÃO DA CAPACIDADE DE VEDAMENTO DA REGIÃO CERVICAL POR MATERIAIS USADOS NA CONFECÇÃO DO TAMPÃO DURANTE O CLAREAMENTO DENTAL ENDÓGENO
}

\author{
THE ASSESSMENT OF THE SEALING CAPACITY OF \\ MATERIALS USED IN THE CONFECTION OF PLUGS \\ FOR THE CERVICAL REGION \\ DURING ENDOGENOUS DENTAL BLEACHING
}

WALISON ARTHUSO VASCONCELLOS ${ }^{1}$ BRUNO ROQUE PATRÍCIO ASSIS ${ }^{1}$ RODRIGO DE CASTRO ALBUQUERQUE ${ }^{2}$

1 Mestrando em Dentística Restauradora pela UFMG

2 Professor da disciplina de Dentística Restauradora da UFMG e da disciplina de Materiais Dentários da Universidade de Itaúna

RESUMO

A reabsorção radicular externa é o principal problema associado ao clareamento endógeno, sendo a confecção de um tampão cervical uma das formas indicadas na sua prevenção. Este estudo se propõe a avaliar a capacidade de 
vedamento da região cervical de diferentes tipos de materiais usados na confecção desse tampão. Foram utilizados 32 incisivos recém extraídos, divididos aleatoriamente em 4 grupos: Grupo I (controle): desobstrução parcial do conduto deixando apenas $1 \mathrm{~mm}$ cervical desobturado; Grupo II: ionômero de vidro modificado por resina; Grupo III: cimento fosfato de zinco; Grupo IV: cimento resinoso. A solução corante constituída de Randomina B a 0,2\% e perborato de sódio foi inserida no interior da câmara pulpar, sendo os corpos de prova submetidos à ciclagem térmica. Os corpos de prova foram lavados, incluídos em resina acrílica, cortados e realizada a leitura. Nos grupos I e IV, grau 0 de infiltração foi observado em 75 \% dos corpos de prova, enquanto nos grupos II e III, 50 \% dos corpos de prova apresentaram respectivamente grau 1 e 2 . Os resultados foram submetidos à análise de variância, apresentando diferença significante, sendo lícito concluir que o grupo I (controle) apresentou o melhor resultado, seguido em segundo pelo grupo IV (cimento resinoso).

Palavras-chave: peróxido de hidrogênio; clareamento; reabsorção radicular

\section{Introdução}

A odontologia, na atualidade, tem a "estética" como um aspecto importante, no qual a beleza é influenciada pelo contorno, forma, simetria, alinhamento e principalmente a cor dos dentes.

O clareamento endógeno tem se tornado um procedimento muito utilizado na prática odontológica e está indicado no tratamento de dentes anteriores despolpados escurecidos. Dentre as principais causas da alteração de cor em dentes despolpados pode-se destacar: presença de materiais restauradores na coroa, hemorragia no interior da câmara pulpar, decomposição de tecidos ou detritos situados no interior da câmara pulpar, medicamentos de uso intracanal e materiais de obturação do canal radicular (DE DEUS, 1992).

Tal procedimento foi empregado pela primeira vez, em 1918, por Abbot (1918), que fez uso do superoxol - denominado na época como peridrol - uma solução de peróxido de hidrogênio a 30 \%, diluído em água, ativado por raios de luz elétrica. Em 1924, Prinz (1924) propôs o clareamento endógeno utilizando uma solução saturada de perborato de sódio e peróxido de hidrogênio, sendo tal solução até hoje utilizada. 
O clareamento endógeno apresenta resultados satisfatórios esteticamente, mas não muito raramente o efeito colateral notado é a reabsorção cervical radicular externa, que pode prejudicar severamente ou até mesmo inviabilizar a permanência do elemento dental na cavidade bucal (HARRINGTON e NATKIN, 1979; MCINERNEY e ZILLICH, 1992; ROTSTEIN et al., 1992; VIEIRA, 1996).

Vários autores (HARRINGTON e NATKIN, 1979; HELLER et al., 1992; KEHOE, 1987; McINERNEY e ZILLICH, 1992; ROTSTEIN et al., 1992) têm mostrado que a reabsorção cervical radicular externa está associada ao calor usado na técnica termocatalítica somado com a ação cáustica do peróxido de hidrogênio. O peróxido de hidrogênio difunde-se, via canalículos dentinários, através da região cervical e penetra pela falha existente, em 10\% dos dentes, na junção cemento-esmalte, atingindo a região do ligamento periodontal (AVNY et al., 1973; HARRINGTON e NATKIN, 1979; KOULAOUZIDOU et al., 1996). Harrington e Natkin, em 1979, relataram 4 casos de reabsorção externa após clareamento endógeno. Desde essa época, passou-se a questionar os motivos da reabsorção radicular cervical externa e maneiras de evitá-la.

A fim de evitar a penetração do peróxido de hidrogênio da região cervical para as regiões do ligamento periodontal, vários autores (LADO et al.,1983; McINERNEY e ZILLICH, 1992; ROTSTEIN et al., 1992; VIEIRA, 1996) recomendam a confecção de um tampão na embocadura do canal radicular. Lado et al., em 1983, após considerações sobre alguns dos fatores que podem levar à reabsorção cervical após clareamento dental, apresentaram a confecção do tampão cervical feito com cimento de policarboxilato como uma alternativa para minimizar aquele processo. Desde então outros materiais têm sido usados para realizar esse tampão cervical, sempre na busca do material que melhor promova o vedamento da região cervical.

Levando-se em consideração a grande importância de se prevenir a possível ocorrência de uma reabsorção externa após a execução de um clareamento dental endógeno, e o importante papel exercido pelo tampão cervical na prevenção desse processo, o propósito deste estudo foi avaliar a capacidade de vedamento da região cervical, de diferentes tipos de materiais usados na confecção do tampão durante o clareamento dental endógeno. 


\section{Material e métodos}

Foram utilizados 32 incisivos inferiores íntegros, recém extraídos e unirradiculares, possuindo apenas um canal radicular. Esses dentes foram previamente analisados sob lupa estereoscópica com aumento de 50 vezes na procura de falhas ou trincas superficiais e armazenados em solução de formalina a $10 \%$ a fim de evitar ressecamento.

A instrumentação e obturação do canal radicular foi feita por um especialista em endodontia. Os canais radiculares foram instrumentados com limas de níquel-titânio com a utilização do aparelho Quantec 2000 (Tycoon) até a lima 8 e posteriormente obturados com cimento Sealer 26 (Dentsply Ind. e Com. Ltda.) e cones de guta percha M e FF (Dentsply Ind. e Com. Ltda.) pela técnica híbrida com condensadores de guta percha Mac Spaden n ${ }^{\circ} 40$.

O excesso dos cones foi cortado no nível amelo-cementário vestibular e feita uma vigorosa condensação vertical. A câmara foi limpa com xilol (Lenzafarm Farmacêutica). A entrada da câmara foi então selada com cimento de óxido de zinco e eugenol Pulpo-Sam (Andrade e Filhos Ltda.) e os dentes armazenados em água destilada.

Os dentes foram preparados com uma broca esférica $n^{\circ} 6$ de haste longa para contra-ângulo (Maillefer-Switzerlend) em baixa rotação. Foi realizada a desobturação parcial do canal radicular penetrando $3 \mathrm{~mm}$ em direção apical.

Os dentes foram divididos aleatoriamente em 4 grupos, de 8 elementos cada, e submetidos aos seguintes tratamentos:

- Grupo I (controle): desobstrução parcial do conduto, deixando apenas $1 \mathrm{~mm}$ cervical desobturado.

- Grupo II: confecção do tampão cervical com o ionômero de vidro modificado por resina denominado Vivaglass-Liner (Vivadent-SchaanLiechtenstein).

- Grupo III: confecção do tampão cervical com cimento fosfato de zinco (S. S. White).

- Grupo IV: confecção do tampão cervical com o cimento resinosoSealer 26. (Dentsply).

Nos grupos II, III e IV, os canais radiculares foram parcialmente desobstruídos, os materiais manipulados segundo recomendações do fabricante e inseridos na região cervical do conduto através de uma seringa Centrix (DFL Ind. e Com. Ltda.). O preenchimento foi feito até o limite 
amelo-cementário vestibular. Excessos de cimento foram removidos com uma broca de aço $\mathrm{n}^{\circ} 6$ até obter-se uma espessura de $2 \mathrm{~mm}$. Colocou-se então uma bolinha de algodão na câmara pulpar e selou-se sua entrada com Pulpo-Sam. Os dentes foram armazenados em água destilada à temperatura ambiente em um frasco plástico com tampa.

O perborato de sódio (Lenzafarm Farmacêutica) recém manipulado foi corado com Randomina B a 0,2\% (SÁ e GABRIELLI, 1979; SPARRIUS e GROSSMAN, 1989; VIEIRA, 1988) (Lenzafarm Farmacêutica). Removeu-se o selamento provisório da entrada da câmara pulpar dos corpos de prova de todos os grupos e, em seguida foi feito o condicionamento ácido da dentina da câmara pulpar com ácido fosfórico a 37 \% gel (Dentsply Ind. e Com. Ltda.) por 15 segundos (ANITUA et al., 1990; BARANTIERI et al., 1993; McINERNEY e ZILLICH, 1992; TITLEY et al., 1988) seguido de lavagem abundante com jato de água por 30 segundos e secagem com jatos de ar. Após esse condicionamento, o agente traçador (denominação dada à mistura do corante com o perborato de sódio) foi colocado no interior da câmara pulpar, que teve sua entrada selada com cimento de óxido de zinco e eugenol, Pulpo-Sam.

Após o selamento da câmara pulpar, aguardou-se 30 minutos e todos os 32 corpos de prova, simultaneamente, foram submetidos à ciclagem térmica seguindo a seguinte técnica (VIEIRA, 1996): os corpos de prova foram colocados em conjunto na máquina de termociclagem (Modelo 521-4 - Nova Ética Ind., Com. e Serv. Ltda.) e submetidos a banhos de 15 segundos, sob temperaturas de $50^{\circ} \mathrm{C}$ e $10^{\circ} \mathrm{C}$ por 100 ciclos.

Após os procedimentos de ciclagem térmica os corpos de prova foram armazenados em estufa (Fanen) durante 7 dias em frascos plásticos com água destilada, mantidos a uma temperatura de $37 \pm 2^{\circ} \mathrm{C}$ (DE PERALTA JR. et al., 1991; HANSEN-BAYLESS e DAVIS, 1992; SMITH et al., 1992).

Decorrido o tempo de armazenamento, os dentes foram lavados em água corrente. Foi removido o selamento da abertura coronária bem como a pasta com o agente traçador e foi realizada a lavagem da câmara pulpar com jatos de água corrente. Em seguida, os dentes foram embutidos em resina acrílica em secções de um tubo de PVC de meia polegada. Essas inclusões foram aparadas e seccionadas longitudinalmente, de mesial para distal, com o auxílio da máquina de corte Isomet 1000, através do disco diamantado (Buehler) à velocidade de 750 rpm com peso de 350 gramas, 
sob irrigação constante com água. Dessa forma, a dentina da região dos 3 mm cervicais do conduto radicular e do limite amelo-cementário ficou exposta para avaliação da penetração do agente traçador.

Utilizou-se a lupa estereoscópica sob aumento de 50 vezes para avaliar visualmente a microinfiltração do perborato de sódio, de acordo com a penetração do agente traçador.

A leitura dos resultados foi executada por 3 examinadores experientes, previamente calibrados. Os corpos de prova apresentaram 4 leituras cada, sendo considerado como escore final o valor mais prevalente nas leituras (Figuras 1 e 2). Os seguintes escores foram utilizados:

0 - Nenhuma penetração do agente traçador entre tampão e dentina

1 - Infiltração do agente traçador até a metade do comprimento total do tampão

2 - Infiltração do agente traçador por toda a extensão do tampão

3 - Infiltração do agente traçador até a base do tampão

Os resultados obtidos foram submetidos à análise estatística e ao Método de Tukey.

Esquema 1: Escores de infiltração

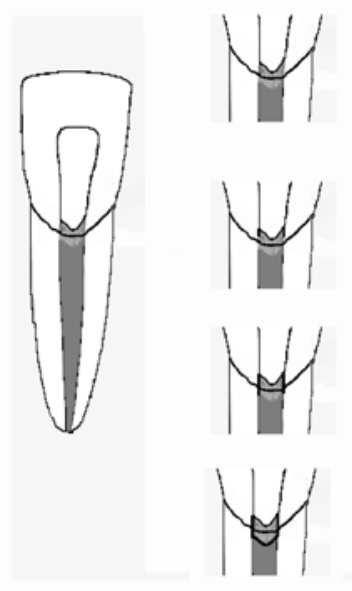

0 - Sem penetração do agente traçador

1 - Infiltração do agente traçador até a metade do comprimento total do tampão

2 - Infiltração do agente traçador por toda a extensão do tampão

3 - Infiltração do agente traçador até a base 

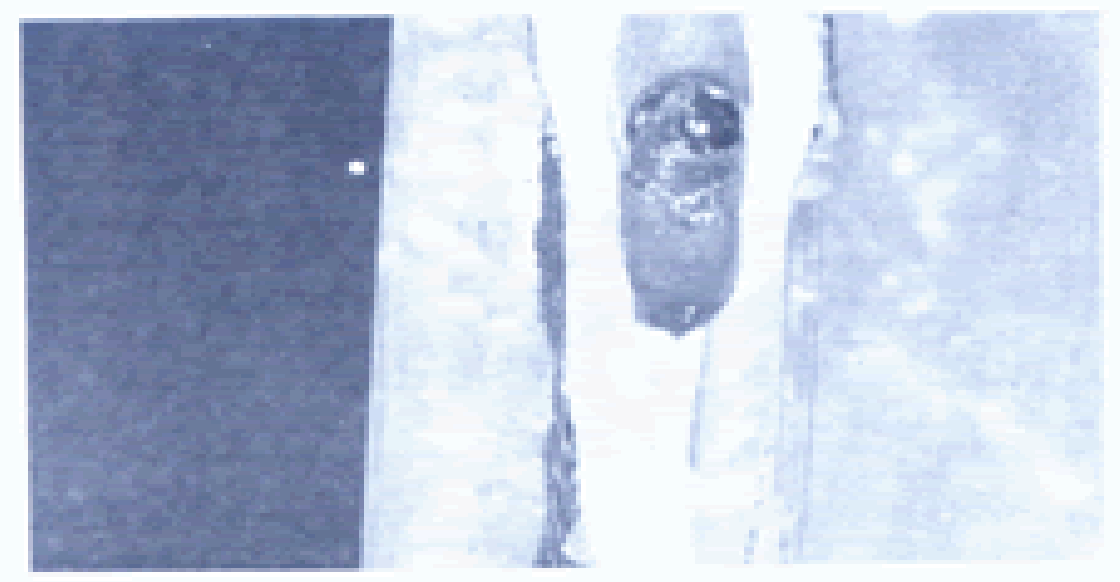

Figura 1 - Corpo de prova representando grau 0 de infiltração (Grupo IV)

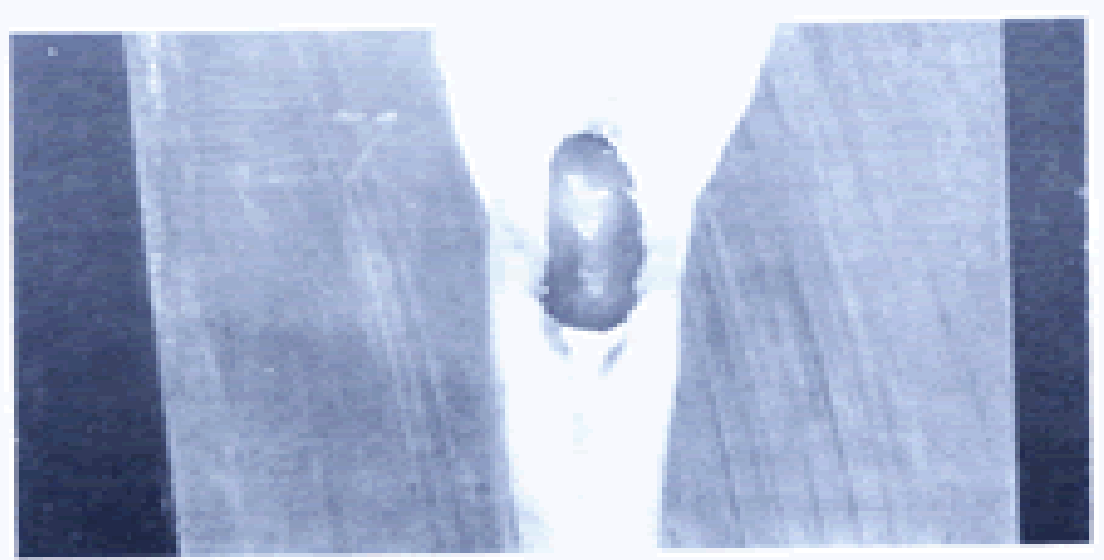

Figura 2 - Corpo de prova representando grau 2 de infiltração (Grupo III)

\section{Resultados}

A avaliação da penetração do corante foi executada para os corpos de prova, estando os resultados expressos na Tabela 1. 
Tabela 1 - Escores de infiltração marginal em função dos grupos experimentais

\begin{tabular}{|c|c|c|c|}
\hline CONTROLE & VIVAGLASS & FOSFATO DE ZINCO & SEALER 26 \\
\hline 0 & 3 & 0 & 0 \\
\hline 0 & 1 & 3 & 3 \\
\hline 0 & 0 & 2 & 0 \\
\hline 1 & 1 & 0 & 1 \\
\hline 0 & 2 & 2 & 0 \\
\hline 0 & 1 & 2 & 0 \\
\hline 0 & 1 & 0 & 0 \\
\hline 1 & 2 & 2 & 0 \\
\hline
\end{tabular}

Os escores individuais foram utilizados para a realização da análise de variância e do Método de Tukey apresentados nas tabelas 2 e 3 respectivamente.

Tabela 2 - Análise de variância

\begin{tabular}{|l|c|c|c|c|}
\hline CAUSAS DE VARIAÇÃO & GL & SQ & QM & F \\
\hline TRATAMENTOS & 3 & 8,25 & 2,75 & 3,05 \\
\hline RESÍDUOS & 28 & 25,25 & 0,90 & \\
\hline TOTAL & 31 & 33,50 & & \\
\hline
\end{tabular}

Para $\alpha=5 \%$, tem-se $F=2,95$ (valor crítico).

Tabela 3 - Valores absolutos da diferença entre os pares de média

\begin{tabular}{|c|c|}
\hline PARES DE MÉDIA & $\begin{array}{c}\text { VALOR ABSOLUTO DA } \\
\text { DIFERENÇA }\end{array}$ \\
\hline GRUPO I X GRUPO II & $1.125^{*}$ \\
\hline GRUPO I X GRUPO III & $1.125^{*}$ \\
\hline GRUPO I X GRUPO IV & 0,25 \\
\hline GRUPO II X GRUPO III & 0 \\
\hline GRUPO II X GRUPO IV & 0.875 \\
\hline GRUPO III X GRUPO IV & 0.875 \\
\hline
\end{tabular}

Para $\alpha=5 \%$, tem-se d.m.s. $=1.17$

*Significante. 
No gráfico 1 é apresentada a média dos escores de infiltração por grupos.

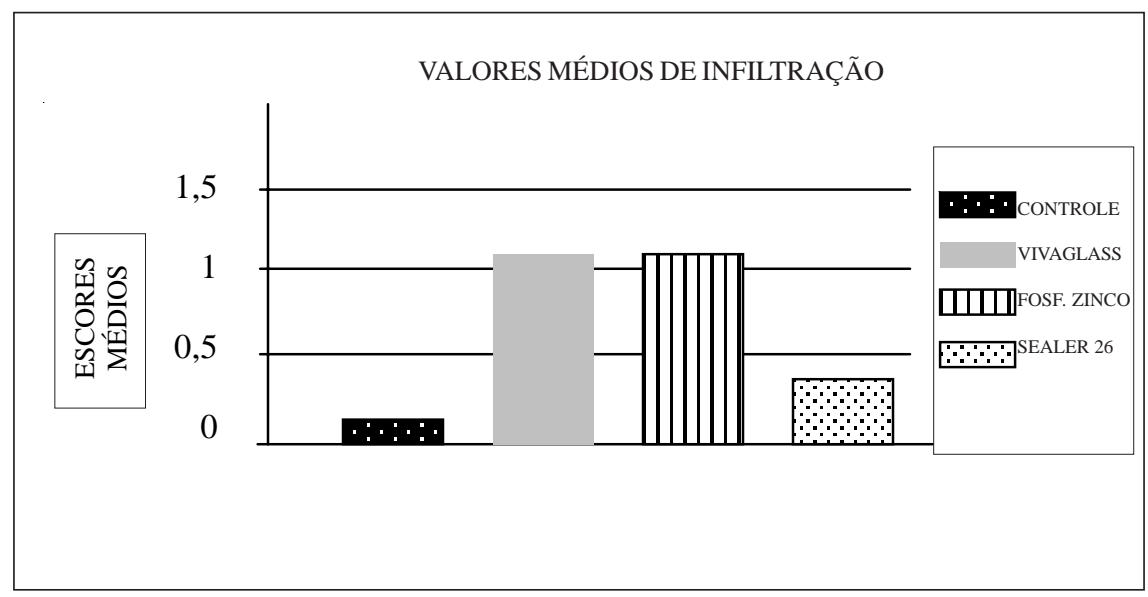

\section{Discussão}

O clareamento endógeno tem se tornado um procedimento muito utilizado na prática odontológica, porém a reabsorção cervical radicular externa associada a tal procedimento é fator de grande importância para os profissionais.

A reabsorção cervical radicular externa está associada ao calor usado na técnica termocatalítica somada com a ação cáustica do peróxido de hidrogênio que se difunde para a região do ligamento periodontal (AVNY et al., 1973; HARRINGTON e NATKIN, 1979; HELLER et al., 1992; KEHOE, 1987; KOULAOUZIDOU et al., 1996; McINERNEY e ZILLICH, 1992; ROTSTEIN et al., 1992).

A confecção de um tampão na embocadura do canal radicular é recomendada a fim de evitar a penetração do peróxido de hidrogênio. Dentre os materiais usados para a confecção do tampão podemos citar: cimento de policarboxilato, cimento de ionômero de vidro, fosfato de zinco e cimento resinoso, dentre outros. 
Os resultados obtidos apresentaram vedamento significativamente melhor para o grupo que não recebeu tampão, ficando o material obturador do canal responsável pelo selamento da região cervical. O grupo tratado com Sealer 26 obteve o segundo melhor resultado. Os grupos nos quais os tampões foram confeccionados com cimento de ionômero de vidro e cimento fosfato de zinco apresentaram elevado grau de infiltração quando comparados com os demais.

Os resultados encontrados para o grupo I estão de acordo com os achados encontrados por Vieira (1996). Tal fato leva-nos a pensar sobre a necessidade de se fazer um tampão, visto o melhor resultado obtido quando da não execução do mesmo. Deve-se ressaltar que os casos que chegam para tratamento clareador já se encontram tratados endodonticamente e que a causa do escurecimento se encontra relacionada, na maioria das vezes, a um tratamento endodôntico inadequado, o que não nos permite confiar nesse tratamento endodôntico para selamento da região cervical.

No que se refere ao grupo tratado com Sealer 26, os resultados obtidos estão de acordo com vários trabalhos que registraram adequado vedamento para esse material (BONETTI FILHO et al., 1995; TANOMARU FILHO, 1999). É importante salientar que a utilização de tal material implica no acréscimo de uma sessão clínica visto que o mesmo apresenta propriedades clínicas aceitáveis apenas 24 horas após sua inserção na cavidade. O grupo tratado com Vivaglass apresentou elevada infiltração o que corrobora os achados de Rotstein et al.(1992) e Bonetti Filho et al. (1995). O grupo fosfato de zinco apresentou elevada infiltração, reafirmando o trabalho de Mac Inerney e Zillich (1992).

Além do material utilizado, outros fatores exercem grande influência na capacidade de vedamento, dentre os quais podemos destacar: a espessura da base protetora e a utilização ou não de calor. Rotstein et al., em 1992, afirmam que todos os materiais testados são igualmente eficazes na prevenção da penetração do peróxido de hidrogênio, quando a espessura da camada da base excede $1 \mathrm{~mm}$.

\section{Conclusão}

Pelos resultados da avaliação experimental parece-nos lícito concluir que:

PUBLICATIO UEPG - Biological and Health Sciences, 6 (1): 29-42, 2000. 
- Nenhum dos grupos testados proporcionou selamento perfeito.

- Os melhores resultados obtidos foram os do grupo I, onde o material obturador usado no tratamento endodôntico foi deixado na região cervical, funcionando como tampão.

- O grupo IV (Sealer 26) teve o segundo melhor resultado, apresentando menor penetração de corante que os grupos II e III.

- Os grupos II e III apresentaram uma infiltração estatisticamente igual entre si, e mostraram-se ineficientes no vedamento da região cervical.

- Novas pesquisas devem ser feitas para se achar um material efetivo no vedamento da região cervical.

Recebido para publicação em 29/6/00.

Aceito para publicação em 16/10/00.

\begin{abstract}
External root resorption is the main problem associated to endogenous bleaching , and the confection of a cervical plug is considered to be the most favorable means of prevention. This research evaluates the sealing capacity of different kinds of materials used in the confection of plugs for the cervical region. Thirty-two incisors, freshly extracted, randomly divided into four groups were used: Group I (control): partial deobstruction of the conduct, deobstructing only $1 \mathrm{~mm}$ of the cervical region; Group II: glass ionomer modified by a resin component; Group III: zinc phosphate cement; Group IV: resinous cement . The colorific solution consisting of $0,2 \%$ of Randomine B and sodium perborate was introduced into the pulp chamber and the test bodies (incisors) were submitted to a thermic cycle. Next, the bodies were washed and enclosed in acrylic resin, then they were cut and analyzed. In groups I and IV a 0 degree of infiltration was found in $75 \%$ of the test bodies, while in groups II and III, 50\% had, respectively, degrees 1 and 2. The results were submitted to a variation analysis and no significant differences were found . It may be concluded that group I (control) had the best results, followed by group IV (resinous cement).
\end{abstract}

Key words: hydrogen peroxide; bleaching; radicular reabsorption 
40

Apoio: FAPEMIG: Fundação de Apoio e Amparo à Pesquisa do Estado de Minas Gerais.

Endereço para contato: Dr. Walison Arthuso Vasconcellos

e-mail: vasconcelloswa@hotmail.com

(31) 374-8063

\section{REFERÊNCIAS}

1 ABBOT, C. H. Bleaching of discolored teeth by means of 30\% perhydrol and electric light rays. J. Allied Dent. Soc., v. 13, p. 259, 1918.

2 ANITUA, E.; ZABALEGUI, B.; GIL, J.; GASCON, F. Internal bleaching of severe tetracycline discolorations: four-year clinical evaluation. Quintessence Int., v. 21, p. 783-788, 1990.

3 AVNY, W. Y.; HEIMAN, G. R.; MADONIA, J. V.; WOOD, N. K.; SMULSON, M. H. Autoradiographic studies of intracanal diffusion of aqueous and camphorated parachlorophenol in endodontics. Oral Surg. Oral Med. O., v. 36, p. 80-9, 1973.

4 BARATIERI, L. N. et al. Clareamento Dental. São Paulo : Quintessence, 1993.

5 BONETTI FILHO, I.; LEONARDO, M. R.; GUIDOTTI, P. C.; LOFREDO, L. C. M. Capacidade seladora de novos cimentos obturadores. RGO., v. 43, n. 4, p. 221-4, 1995

6 DE DEUS, Q. D. Clareamento de dentes com alteração de cor. In: DE DEUS, Q. D. Endodontia. 5. ed. Rio de Janeiro : Medsi, p. 627-40, 1992.

7 DE PERALTA JR., A. A. et al. Apical leakage of bleaching agents through an intermediate dental base material. Gen. Dent., Nov.-Dec., 1991.

8 HANSEN-BAYLESS, J.; DAVIS, R. Sealing ability of two intermediate restorative materials in bleached teeth. Am. J. Dent., v. 5, p. 151-4, 1992.

9 HARRINGTON, G. W.; NATKIN, E. External resorption associated with bleaching of pulpless teeth. J. Endodont., v. 5, p. 344-8, 1979.

10 HELLER, D.; SKRIBER, J.; LIN, L. M. Effect of intracoronal bleaching on external cervical root resorption. Amer. Assoc. Endod., v. 18, n. 4, Apr., 1992.

PUBLICATIO UEPG - Biological and Health Sciences, 6 (1): 29-42, 2000. 
11 KEHOE, J. C. pH reversal following in vitro bleaching of pulpless teeth. $\mathbf{J}$. Endodont., v. 13, p. 6-9, 1987.

12 KOULAOUZIDOU, E. ; LAMBRIANIDIS, T.; BELTES, P.; LYROUDIA, K.; PAPADOPOULOS, C. Role of cementoenamel junction on the radicular penetration of 30\% hydrogen peroxide during intracoronal bleaching in vitro. Endod. Dent. Traumatol., n. 12, p. 146-50, 1996.

13 LADO, E. A.; STANLEY, H. R.; WEISMAN, M. I. Cervical resorption in bleaching teeth. Oral Surg. Oral Med. O., v. 55, p. 78-80, 1983.

14 MacISAAC, A. M.; HOEN, C. M. Intracoronal Bleaching: concerns and considerations. J. Endodont., v. 20, n. 6, p. 304-6, 1994.

15 McINERNEY, S.; ZILLICH, R. Evaluation of internal sealing of three materials. J. Endodont., v. 18, p. 376-8, 1992.

16 PRINZ, H. Recent improvements in tooth bleaching. Dent. Cosm., v. 66, p. 558, 1924.

17 ROTSTEIN, I.; FRIEDMAN, S.; MOR, C.; KATZNELSON, J.; SOMMER, M.; BAB, I. Histological characterization of bleaching-induced external root resorption in dogs. J. Endodont., p. 436-41, Sep. 1991.

18 ROTSTEIN, I.; ZALKIND, M.; MOR, C.; TARABEAH, A.; FRIEDMAN, S. In vitro efficacy of sodium perborate preparations used for intracoronal bleaching of discolored nonvital teeth. Endod. Dent. Traumatol., v. 7, p. 177-80, Aug. 1991.

19 ROTSTEIN, I.; FRIEDMAN, S. Ph variation among materials used for intracoronal bleaching. J. Endodont., v. 17, p. 376-9, 1991.

20 ROTSTEIN, I.; MOR, C.; FRIEDMAN, S. Prognosis of intracoronal bleaching with Sodium Perborate preparations in vitro: 1- Year study. J. Endodont., v. 19, p. 10-2, 1993.

21 ROTSTEIN, I.; TOREK, Y.; MISGAV, R. Effect of cementum defects on radicular penetration of 30\% H2O2 during intracoronal bleaching. J. Endodont., v. 17, p. 230-3, 1991.

22 ROTSTEIN, I.; ZYSKIND, D.; LEWINSTEIN, I.; BAMBERGER, N. Effect of different protective base materials on hidrogen peroxide leakage during intracoronal bleaching in vitro. J. Endodont., v. 18, p. 114-7, 1992. 
23 SÁ, D. N.; GABRIELLI, F. Estudo da infiltração marginal em restaurações com amálgama. Efeito de liga, verniz e brunidura. Rev. Farm. Odontol., Ribeirão Preto, v. 16, p. 53-62, 1979.

24 SMITH, J. J.; CUNNINGHAM, C. J.; MONTGOMERY, S. Cervical canal leakage after internal bleaching procedures. J. Endodont., v. 18, p. 476-81, Oct. 1992.

25 SPARRIUS, O.; GROSSMAN, E. S. Marginal leakage of composite resin restorations in combination with dentinal and enamel bonding agents. J. Prosthet. Dent., v. 61, p. 678-84, 1989.

26 TANOMARU FILHO, M. Capacidade seladora do Sealer 26 e AH plus em obturações retrógradas. Rev. Paul. Odont., n. 1, p. 34-6, 1999.

27 TITLEY, K.; TORNEK, C. D.; SMITH, D. D. Effect of concentrated hidrogen peroxide solution on the surface morphology of cut human dentin. Endod. Dent. Traumatol., v. 4, p. 32-6, 1988.

28 VIEIRA, L. C. C. Avaliação da infiltração marginal nas margens de esmalte e cemento em cavidades de classe II MOD restauradas com sistemas adesivos. Bauru, 1988. Tese (Doutorado) - Faculdade de Odontologia, Universidade de São Paulo.

29 VIEIRA, P. A. A. Avaliação da capacidade de selamento da região cervical de materiais usados na confeç̧ão do tampão cervical durante o clareamento dental intracoronário. Araraquara, 1996. Tese (Doutorado) - Faculdade de Odontologia de Araraquara - Unesp. 